\title{
An Open Source Based Application for Integration and Sharing of Multi-modal Cardiac Image Data in a Heterogeneous Environment
}

\author{
P Marcheschi, V Positano, EM Ferdeghini, A Mazzarisi, A Benassi \\ CNR Institute of Clinical Physiology, Pisa, Italy
}

\begin{abstract}
DICOM format has been recognized as the de facto standard for storage, transferring and sharing of cardiac images along different modalities like Magnetic Resonance Imaging (MRI), Nuclear Medicine, Computer Tomography (CT), Digital Angiography (XA), Digital Radiology. However, in many medical environments there is a large need to have cardiac images available in formats (i.e. GIF, BMP or JPEG) that are compatible with widely used office automation applications. The here proposed system for multi modality image sharing and conversion is based on both the standard DICOM communication protocol and the Common Internet File System (CIFS) protocol. DICOM images from any DICOM compliant station can be transferred to a dedicated DICOM server, which has been implemented using the freely available DCMTK DICOM toolkit from Kuratorium OFFIS. An automatic procedure converts DICOM images into the desired image format, creating at the same time a file tree that allows fast and easy retrieval of image data. The entire proposed framework is implemented using Open Source software with large advantages in terms of cost reduction and software flexibility and robustness.
\end{abstract}

\section{Introduction}

The advent of the production of radiological digital images has undoubtedly changed the way of working of the physician with a radical change of its habits due to the use of editing tools and video refertation. These are necessary steps in order to produce the documentation of the patient, for its checkout or the relative follow-up. By now DICOM it is the standard for the transmission and the interpretation of images in medical environment, but the compilation of reports or presentations is undoubtedly done by the physician by standard Office Automation tools, as Microsoft Word $\AA$ or PowerPoint $\AA$. In fact, almost all the programs that allow reading and visualization of DICOM images introduce a menu for the conversion of DICOM data in different digital formats, as jpeg, bmp or tiff. A non exhaustive list of situations, requiring these kinds of image format, includes the integration of images in scientific presentation and documents, as well as into computer-based reporting systems, and into available HIS (Hospital Information System). We propose an application that is able to meet the demands of the physicians and to face the interconnecting problems of diagnostic modalities available in a modern cardiologic diagnostic centre.

Every diagnostic modality that understands in its own software endowment the DICOM standard, allows sending toward a DICOM Server a selection of images selected by the operator. Therefore is possible to exploit this possibility offered by such equipments to transfer the required material for the consultation in such a way that the modality sees a normal DICOM server on the net. As it regards the receiving application, it behaves as a service class provider, in degree to accept images from a service class user for the DICOM service class of storage.

The procedure was developed in Perl programming language. The procedure automatically checks the arrival of new images, extracts demographics information from each of them, converts image data in the needed format and copies the converted images into the appropriate path. The created directory hierarchy is shared by CIFS protocol by means of a SAMBA server. Consequently, the image data can be accessed by any Windows/MacOS/Linux based PCs in the local network. Data access can be filtered by means of the SAMBA security protocol. The whole system runs on standard PC with LINUX operative system. It is important to notice that the system has been totally realized using open source technology, allowing the development of a really low-cost efficient application. The described application was deployed and tested in a clinical environment allowing sharing of cardiac images acquired in MRI and XA modalities, in the local area network.

\section{Open source software}

The basic idea behind open source is quite simple: when programmers can read, redistribute, and modify the source code for a piece of software, the software evolves. Because a large number of developers are involved, the speed and efficiency in improving and adapting the software and in fixing software bugs can results higher respect to conventional software development [1]. Open source doesn't just mean access to the source code. The distribution terms of open-source software must comply with the following criteria [2]: 
1. The license shall not restrict any party from selling or giving away the software as a component of an aggregate software distribution containing programs from several different sources. The license shall not require a royalty or other fee for such sale.

2. The program must include source code, and must allow distribution in source code as well as compiled form.

3. The license must allow modifications and derived works, and must allow them to be distributed under the same terms as the license of the original software.

These criteria will preserve the features of the original license during software dissemination. Open Source approach is of course especially suitable for research institution allowing the knowledge sharing preserving at the same time the intellectual property of the realized applications. Moreover, because software bugs are immediately corrected by developers community, the use of Open Source software can improve the system security [3]. The use of Open Source approach is encouraged by several government documents, in Italy [4] and in other countries.

\section{Open source based server}

The server for medical images receiving, conversion and sharing is based on Linux Operative System. The storage in Linux environment has been realized with Raid 1 technology that is able to avoid loss of data in case of failure of one of the two Hard disks.

The Linux file system is the ext3 journaled, that is able to avoid any data loss originated from unexpected reboots, unexpected power failure and system lock-ups. In fact, in standard filesystems the previous described occurrences can result in significant corruption of recently-modified data. Ext3, besides, gets rid of the period of wait related to the check of the filesystem, owing to the fact that it exists a journal that holds trace of all the accesses to the disk from the operating system. The system is protected by an integrated firewall subsystem based on Iptables [5], a packet selection system allowing a total protection also in absence of the firewall appointed to the protection of the functional island.

\subsection{System overview}

All the applications running on the previous described server were realized using only open source programming tools. These are Perl, and several DICOM tools such as DCMTK DICOM toolkit from Kuratorium OFFIS and D. Clunie dicomtools.

The application server can automatically receive images from any DICOM compatible device, acting as a DICOM SCP (Service Class Provider) for the storage service [6]. The application that attends redistribution service, on receiving DICOM files from the diagnostic modality, completes the followings footsteps, as depicted in Figure 1:

1. Extracts clinical information from each DICOM file;

2. Prepares the directories in which to save the converted images, and the original DICOM files;

3. Convert DICOM into the appropriate format (e.g. jpeg) taking into account the appropriate $\mathrm{W} / \mathrm{L}$ (Window / Level) for that kind of image;

4. Store a copy of the original DICOM files in a separate directory;

5. Pass the control again to the Service class provider to be able to satisfy new requests.

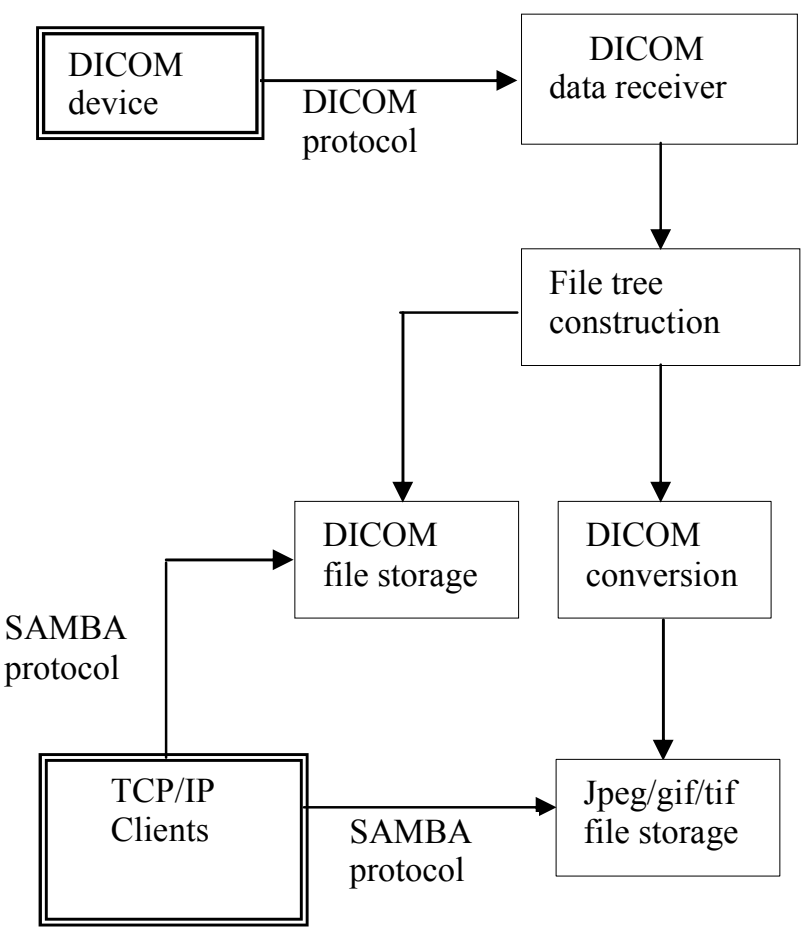

Figure 1: Flow chart for the server activity.

The DICOM and image files become available through a network connection by using a SAMBA protocol server running on the Linux system.

\subsection{File tree construction}

The image data are organized in order to match the requirement of the medical users. Each acquisition should be differentiated, including acquisition done on the same patient in different days. Along each acquisition, single image transmitted by the user from the DICOM devices have to unequivocally be identified. In order to fit these requirements, the realized application extract from DICOM files some clinical information, as the patient 
name, the exam date, the series name, the series and the image number. The patent name and exam data are used to construct a directory in which all images will be stored. Each image will be identified combining the series name, that usually contain some information about the acquisition protocol, the series number and the image number that will unequivocally identify the image. Figure 2 shows the file tree related to a volunteer created from the proposed application. The data are related to a Magnetic Resonance exam.

\begin{tabular}{|l|l|l|l|l|}
\hline $\begin{array}{l}\text { Patient } \\
\text { Name }\end{array}$ & $\begin{array}{l}\text { Exam } \\
\text { date }\end{array}$ & Series Name & $\begin{array}{l}\text { Series } \\
\text { Number }\end{array}$ & $\begin{array}{l}\text { Image } \\
\text { Number }\end{array}$ \\
\hline Cinc Man & 10 Jan 03 & Del-Ench & 2 & 1 \\
\hline Cinc Man & 10 Jan 03 & Del-Ench & 2 & 2 \\
\hline Cinc Man & 10 Jan 03 & Localizer & 1 & 8 \\
\hline Cinc Man & 10 Jan 03 & FIESTA & 4 & 12 \\
\hline Cinc Man & 10 Jan 03 & FIESTA & 4 & 30 \\
\hline Cinc Man & 10 Jan 03 & Screen save & 132 & 1 \\
\hline
\end{tabular}

\section{CINCMAN-20031001}

\begin{tabular}{|ll} 
& Del-Ench-002-001.jpg \\
& Del-Ench-002-002.jpg \\
& FIESTA-004-012.jpg \\
& FIESTA-004-030.jpg \\
& localizer-001-08.jpg \\
\hline & Screensave-132-001.jpg
\end{tabular}

Figure 2: File tree as produced by Perl based application.

The same methodology is followed to create a file tree that will store DICOM images. Original DICOM files will be renamed following the same criteria adopted for the jpeg/tiff/gif format. So that, at the end of the previous described procedure, two directories will be created in two separate folders, the one containing converted images and the other one containing the DICOM ones.

\subsection{Image conversion}

The image conversion feature uses the open source package: DCMTK DICOM toolkit from Kuratorium OFFIS tool [7]. An important issue in DICOM images conversion is about the windowing level. In fact, DICOM format supports 16 bits image format, while image formats as jpeg, tiff or gif are often limited to 8 bit representation.

Rough conversion can consequently leads to an unacceptable loss of information. The Perl application is tuned in order to automatically equalize the image levels distribution providing good quality image. In particular, the application can read the image modality from DICOM header and adapt the conversion to the present modality. Figure 3 shows an example of a rough and adaptive conversion performed on a magnetic resonance image of the hearth.
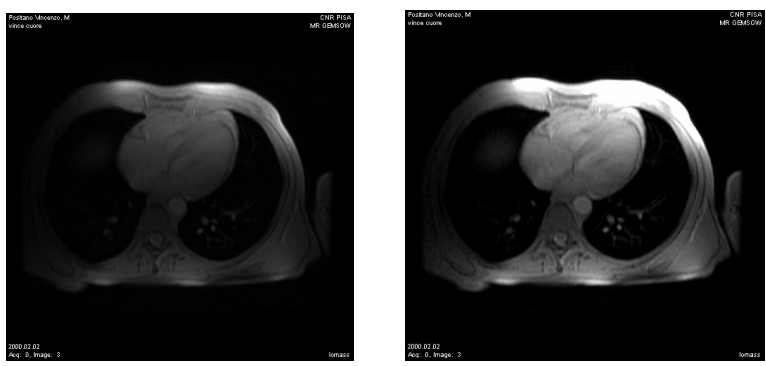

Figure 3: Example of rough (left) and adapted (right) conversion of MR heart image. Adaptive conversion is able to preserve image information.

\subsection{File sharing}

The file tree created in the previous described step should be available across a local area network (LAN) in order to allow easy access to medical users. In order to accomplish this issue, we choose to share data by the Common Internet File System (CIFS), implemented in the well-known SAMBA package $[8,9]$. SAMBA package allows file sharing between different operative systems as LINUX, UNIX, Windows and MacOs. Directories created on the Linux server appear as public folders on "network resources" on a Windows based machine, allowing medical users to access shared data in familiar and effective way. In fact, the user can copy the image data in his private directories or use them whit the favourite application with a "double click" approach.

Samba security should not be the principal way to secure data on a LAN. A hardware firewall is strongly suggested to protect sensible data on the LAN from external unauthorized accesses. Moreover, the Linux system is strengthened by the use of an integrated firewall subsystem based on IPtables, a packet selection system.

However, SAMBA access control can be effectively used to restrict client access, while the samba password security option permits to assign to selected users access to the shared file system.

\section{Result and conclusions}

The previous described system was realized and tested in the Cardiac Magnetic Resonance Laboratory at the CNR Institute of Clinical Physiology in Pisa, Italy.

In the MRI laboratory four DICOM compatible devices are available, daily used for MR image visualization and processing. A local area network (100 $\mathrm{Mb} / \mathrm{sec}$ speed ) connect the DICOM devices with about ten personal computer, eight based on Windows NT/2000 operative system and two based on MacOs, used by cardiologists and technicians. The system was used to prepare well organized image data set for sharing with 
other cardiologists, to use MRI images for preparing scientific papers and presentations and to insert image data in computer based clinical referts.

The proposed system was tested during about six months showing a good robustness together with a noticeable effectiveness.

In conclusion, we can state that a system for multi modality image sharing and conversion, based on both standard DICOM communication protocol and Common Internet File System (CIFS) protocol can simplify the work of medical doctors and technician in a modern cardiologic laboratory. The proposed system allows transferring images from any DICOM compliant station to a public area accessible in intuitive way for laboratory people. An automatic procedure converts DICOM images in the needed image format, creating at the same time a file tree that allows fast and easy retrieval of image data.

The whole system runs on standard PC with LINUX operative system. It is important to notice that the system was totally realized using open source technology, allowing the development of an effective low-cost application.

\section{References}

[1] Perkins G. Culture clash and the road to world domination. IEEE Software, 1999;16(1):80 -84.

[2] Stallman RM. Free Software, Free Society: Selected Essays of Richard M. Stallman. Joshua Gay. 2002.

[3] Witten B, Landwehr C, Caloyannides M. Does open source improve system security? IEEE Software 2001;18(5):57 61.

[4] Ministro per le innovazioni e le tecnologie - comunicato del 26/06/2003. Available at URL:www.innovazione .gov.it/ita/comunicati/2003_06_12.shtml. Accessed Sept 2003 (Italian).

[5] Bauer MD. Building Secure Servers with Linux. O'Reilly \& Associates, 2002.

[6] DICOM homepage. Available at URL: http: //www.medical.nema.org/. Accessed Sept 2003.

[7] OFFIS | PROJECTS | DICOM. Available at URL:http:// www.offis.de/projekte/ig/dicom/index e.php. Accessed Sept 2003.

[8] Ts J, Eckstein R, Collier-Brown D. Using Samba, 2nd ed., O'Reilly \& Associates, 2003.

[9] Samba web pages. Available at http://www.samba.org/. Accessed Sept 2003.

Address for correspondence.

P. Marcheschi

CNR Institute of Clinical Physiology

CNR Research Area - Via G. Moruzzi 1

56124 PISA (ITALY)

E-mail address: paolo@ifc.cnr.it 